\title{
Degrees of Freedom of Two-Cluster MIMO Multiway Relay Interference Channels Using Blind Interference Neutralization
}

\author{
Bowei Zhang*, Wenjiang Feng, Tingting Dong and Yina Deng \\ College of Communication Engineering, Chongqing University \\ Chongqing, China, 400044 \\ [e-mail: \{20101213140, fengwj, 20131213067, 20131202006t\}@cqu.edu.cn]
}

*Corresponding author: Bowei Zhang

Received August 4, 2015; revised October 11, 2015; accepted October 29, 2015; published January 31, 2016

\begin{abstract}
In this paper, we investigate the degrees of freedom (DoF) of a two-cluster multiple-input multiple-output (MIMO) multiway relay interference channel (mRIC), where there are two relays and two users per cluster. In this channel, users within the same cluster exchange messages among themselves with the help of two relays. We first obtain the DoF upper bound of the considered MIMO mRIC based on cut-set bound. Then, we propose a novel transmission strategy, blind interference neutralization (BIN), to approach the DoF upper bound. This new method utilizes the overheard information at two relays and focuses on the beamforming matrix designs at two relays so that the channel state information (CSI) at users is not required. Through theoretical analysis and numerical simulations, we show that the DoF upper bound can be obtained by using the BIN scheme. From simulation results, we show that the proposed BIN scheme can provide significant performance gain over the conventional time division multiple access (TDMA) scheme in terms of DoF. In addition, we show that the BIN scheme is a superior approach to the existing signal space alignment (SSA) schemes for the considered mRIC.
\end{abstract}

Keywords: multiway relay, interference channel, degrees of freedom, multiple-input multiple-output, interference neutralization, signal space alignment 


\section{Introduction}

Recently, wireless relay channels have been studied intensively because relay technique can be served as a powerful approach to improve the throughput and system capacity of wireless networks. The classic one-way relay systems require four time slots to accomplish bidirectional communication, whereas two-way relay systems only need two time slots. Compared to one-way relay system, several studies [1-3] have shown that the sum rate performance of the two-way relay system increases significantly. In two-way relay channels, concurrent transmission gives rise to sum rate gain due to the knowledge of side information. Roughly speaking, when two users simultaneously transmit signals to the intermediate relay at the multiple access (MAC) phase, the relay node can utilize the physical-layer network coding [4-6] to decode a combination of the signals transmitted from the two users. Then, the relay broadcasts a network-coded message at the broadcast (BC) phase. By exploiting the knowledge of side information, each user can eliminate the self-interference and decode the desired message.

The two-way relay channel can naturally be generalized to the multiway relay channel (mRC), where multiple users want to exchange information with the help of an intermediate relay node. Recently, the general mRC model has been proposed in [7], where there is one relay node, arbitrary number of clusters and arbitrary number of users per cluster. Nevertheless, the exact capacity is difficult to be determined for the general mRC because the channel model is very complex. The degrees of freedom (DoF) known as the capacity pre-log or the multiplexing gain can characterize the approximate capacity of wireless networks in high signal-to-noise ratio (SNR) regime. Therefore, DoF is an important metric to measure the performance of wireless networks. In order to obtain the optimal DoF of the general mRC, we need to determine the maximum number of interference-free signals. Due to the superposition nature of the wireless medium, the relay node in the mRC needs to retain the signals transmitted from all users. The main reason is that the interfering signal for one user is probably the desired signal for the other users. Therefore, the sophisticated strategies for the general mRC should be designed to deal with multiple signals at the relay so that the desired signal can be extracted from the network-coded message at each user.

\subsection{Related Work}

Based on the idea of interference alignment (IA) [8-10], a similar concept, signal space alignment (SSA) was first proposed in [11] to investigate the maximum achievable DoF for the multiple-input multiple-output (MIMO) Y channel (a specific case of the mRC with three users in one cluster), where three users want to communicate with each other via a relay. The essence of SSA lies in aligning the useful signals to maximize the available spatial dimension at the relay and utilizing network coding to achieve the optimal DoF. The idea of SSA was extended to the $K$-user Y channel in [12]. By exploiting the SSA method, the authors in [13, 14] studied the achievable DoF of the asymmetric (arbitrary number of antennas at users) and symmetric (equal number of antennas at users) mRCs, respectively. In [15], Wang et al. proposed a systematic SSA scheme to analyze the DoF of the symmetric MIMO mRC and showed that the DoF upper bound can be achieved in a wider range than the conventional SSA.

Interference neutralization (IN), which is also known as distributed interference cancelling or distributed zero forcing, has been applied in interference networks and wireless relay networks [16-23]. Aligned interference neutralization (AIN) was first introduced by Tian et al. 
[16] to investigate the DoF of the two-hop interference network. By using IA and interference cancellation, the authors in [17] achieved a same DoF and a higher diversity gain compared to the scheme in [8]. The authors in [18] studied the DoF of the three-user MIMO interference channel with a cognitive relay by using AIN and showed that a higher DoF than the scheme in [10] can be obtained. By using the AIN scheme, the authors in [19] studied the DoF of two-user wireless networks with an instantaneous relay and showed that the instantaneous relay can increase the DoF in wireless networks. Subsequently, the idea of AIN was extended to the three-user MIMO interference channel with an instantaneous relay in [20]. By utilizing interference shaping and neutralization (ISN), the authors in [21] obtained the same result with the scheme in [16] without any cooperation at users. The ISN scheme is more practicable than the AIN scheme, because it does not require channel state information (CSI) at users. The problem of CSI accuracy was investigated in the multi-cell network [22]. In [23], the feasibility of IN was analyzed for the multi-cell MIMO interference broadcast channel with multiple relays and users. Based on the feasibility analysis of IN in [23], the authors in [24] studied the feasibility of IN in the two-user MIMO interference channel with an instantaneous relay. However, the DoF achievability in [23] and [24] is still an open problem.

\subsection{Motivation}

The mRC model with a single relay can be used to model a variety of communication scenarios. For example: ad hoc networks with one central controller and cellular networks with one base station (BS) where the BS serves as a relay to enable communication between users. However, the data transmission via a single relay is not suitable when multiple relays can be utilized to obtain a higher DoF. For example, there are two relays and each relay serves users of one cluster. If all users simultaneously transmit signals to two relays in the same frequency band, each relay receives the inter-cluster interference (ICI). Therefore, the conventional mRC model is inappropriate for this scenario. To address this problem, we propose a new network information flow model called MIMO multiway relay interference channels (mRIC), where multiple mRCs are simultaneously operating in the same frequency band. It is significant to investigate the achievable DoF of the mRIC because the mRIC can be considered as the combination of multiple mRCs and the DoF achievability of the mRIC remains an open problem. As a first step, we consider a two-cluster mRIC, where there are two relays, two users per cluster. Studying the DoF of the considered mRIC can provide valuable insights about the optimal transmission design for the general mRIC.

\subsection{Main Contributions}

In this paper, we aim to study the achievable DoF of the two-cluster MIMO mRIC with two relay nodes and two users per cluster, where each relay node serves users of one cluster. If all users simultaneously transmit their own signals in the same frequency band in the mRIC, two relays can overhear the information transmitted from the interfering users. The key is how to effectively exploit the overheard information to achieve a higher DoF. To this end, we propose a blind interference neutralization (BIN) scheme to investigate the achievable DoF of the considered mRIC. The BIN scheme can utilize the overheard information at two relays to eliminate ICI. The main contributions and results of this work are summarized as follows:

1. To investigate the DoF of the addressed scenario, the DoF upper bound is first obtained based on cut-set bound. Subsequently, based on the proposed BIN scheme, we confirm the achievability of the DoF upper bound. The DoF analysis also reveals that asymmetry in the number of antennas at users can be utilized to facilitate the BIN scheme. In addition, we show that the BIN scheme can relieve the pressure on each user, because it does not require 
cooperation at users.

2. From the simulation results, we verify that the BIN scheme can provide significant DoF gain over the conventional time division multiple access with network-coding (TDMA-NC) in [14]. For the asymmetric two-cluster MIMO mRIC, we show that the BIN scheme can obtain a higher DoF than the SSA scheme in [13] when the SSA cannot obtain the optimal achievable DoF. For the symmetric two-cluster MIMO mRIC, we show that the BIN scheme can obtain the DoF upper bound in a wider range than the SSA schemes in [14] and [15].

The rest of this paper is organized as follows. In Section 2, we introduce the system model. In Section 3, a DoF upper bound is described as the benchmark of the transmission design. In Section 4, the achievable DoF of the considered MIMO mRIC is investigated and the achievability of the DoF upper bound is proved. The numerical simulations are given in Section 5. Finally, this paper is concluded in Section 6.

Notation: Scalars are denoted by lowercase regular letters, vectors by lowercase bold letters, and matrices by uppercase bold letters. The major matrix symbols to be used in this paper are listed in Table 1.

Table 1. Major matrix symbols

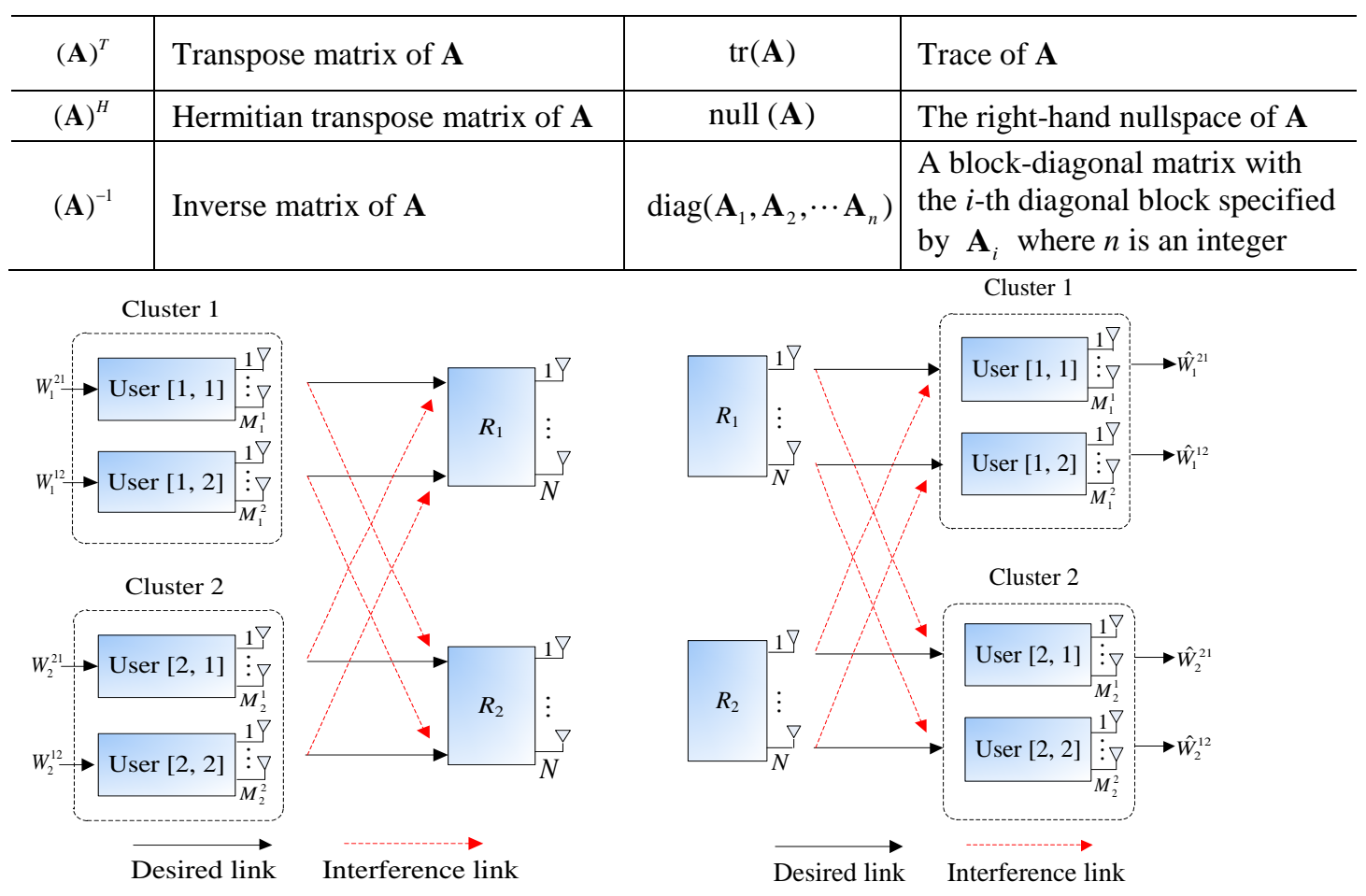

(a) MAC phase

(b) BC phase

Fig. 1. The two-cluster MIMO mRIC with two users per cluster.

\section{System model}

We describe a system model for the two-cluster MIMO mRIC with two relay nodes and two users per cluster as shown in Fig. 1, where relay $R_{1}$ serves users in cluster 1 and relay $R_{2}$ 
serves users in cluster 2. In this channel, all nodes exchange messages in the same frequency band and the users within the same cluster exchange messages among themselves with the help of two relays. At the multiple access (MAC) phase, all users simultaneously transmit signals to two relays. Each relay receives the desired signals transmitted from the serving users and the ICI transmitted from the interfering users. At the broadcast (BC) phase, two relays broadcast the proceed signals to all users. For notation convenience, we refer to the $k$-th user in the $l$-th cluster as user $[l, k]$. User $[l, k](l, k \in\{1,2\})$ is assumed to have $M_{l}^{k}$ antennas and relay $R_{i} \quad(i \in\{1,2\})$ is assumed to have $N$ antennas. Without loss of generality, we assume that, $M_{l}^{1} \geq M_{l}^{2}$ and $M_{1}^{2} \geq M_{2}^{2}$. User $[l, k]$ sends a message $W_{l}^{k^{\prime k}}\left(\forall k^{\prime} \neq k\right)$ to the other users in the $l$-th cluster, i.e., user [1,1] and user [1,2] exchange information $W_{1}^{21}$ and $W_{1}^{12}$ with each other, while user [2,1] and user [2,2] exchange information $W_{2}^{21}$ and $W_{2}^{12}$ with each other.

It is assumed that the direct paths between users are ignored and users can only communicate via the relay. All nodes in this network are assumed to be full-duplex ${ }^{1}$, which implies that all nodes can transmit and receive simultaneously. All the DoF results obtained in this paper directly hold for the half-duplex communication by including a multiplicative factor of $\frac{1}{2}$. The data transmission contains two phases, i.e., the MAC phase and BC phase. Note that the two phases can simultaneously proceed in full-duplex communication.

At the MAC phase, all users simultaneously transmit signals to two relays without precoding. The received signal vector at relay $R_{i}$ is given by

$$
\mathbf{Y}^{R_{i}}=\sum_{l=1}^{2} \sum_{k=1}^{2} \mathbf{H}_{l}^{R_{i}, k} \mathbf{X}_{l}^{k}+\mathbf{Z}^{R_{i}}, \quad i \in\{1,2\}
$$

where $\mathbf{Z}^{R_{i}}$ denotes the $N \times 1$ additive white Gaussian noise (AWGN) vector with zero mean and unit variance at relay $R_{i}, \mathbf{X}_{l}^{k}$ denotes the $M_{l}^{k} \times 1$ transmit signal vector at user $[l, k] . \mathbf{H}_{l}^{R_{i}, k}$ denotes the $N \times M_{l}^{k}$ channel matrix from user $[l, k]$ to relay $R_{i}$. The transmit signal $\mathbf{X}_{l}^{[k]}$ at user $[l, k]$ satisfies transmit power constraint of

$$
\operatorname{tr}\left[\mathbf{X}_{l}^{k}\left(\mathbf{X}_{l}^{k}\right)^{H}\right] \leq P_{l}^{[k]}, k, l \in\{1,2\}
$$

where $P_{l}^{[k]}$ is the maximum transmission power allowed at user $[l, k]$.

After the MAC phase, each relay $R_{i}$ generates the new transmit signal $\mathbf{X}^{R_{i}}=\mathbf{W}^{i} \mathbf{Y}^{R_{i}}, i \in\{1,2\} . \mathbf{W}^{i}$ indicates the linear beamforming matrix at relay $R_{i}$ with the size of $N \times N$. The transmit signal $\mathrm{X}^{R_{i}}$ satisfies the relay power constraint of

$$
\operatorname{tr}\left[\mathbf{X}^{R_{i}}\left(\mathbf{X}^{R_{i}}\right)^{H}\right] \leq P_{R_{i}}, \quad i \in\{1,2\}
$$

where $P_{R_{i}}$ is the maximum transmission power allowed at relay $R_{i}$.

At the BC phase, each relay broadcasts the processed signal to all users. The received signal at user $[l, k]$ is given by

${ }^{1}$ We assume perfect full-duplex operation in this paper. Therefore, there is no residual loop-back interference at each node. 


$$
\mathbf{Y}_{l}^{k}=\sum_{i=1}^{2} \mathbf{H}_{l}^{k, R_{i}} \mathbf{X}^{R_{i}}+\mathbf{N}_{l}^{k}, k, l \in\{1,2\}
$$

where $\mathbf{N}_{l}^{k}$ represents the AWGN vector with zero mean and unit variance at user $[l, k], \mathbf{X}^{R_{1}}$ denotes the $N \times 1$ transmit signal vector at relay $R_{i}, \mathbf{H}_{l}^{k, R_{i}}$ denotes $M_{l}^{k} \times N$ channel matrix from relay $R_{i}$ to user $[l, k]$. It is assumed that the entries of the channel matrices are drawn independently from a continuous distribution, which makes that the channel matrices are full rank almost surely. We also assume that channel state information (CSI) is perfectly known at two relays.

It is assumed that $P_{l}^{[k]}=P_{R_{i}}=P(i, k, l \in\{1,2\})$ and the transmitted power at all nodes is $P$. Since we assume the noise is i.i.d zero-mean complex Gaussian random variables with unit variance, the average received SNR of each link is $P$. The achievable rate $R_{l}^{k^{k}}$ (SNR) of message $W_{l}^{k^{\prime} k}$ is a function of SNR, which is defined as the channel capacity from user $[l, k]$ to user $\left[l, k^{\prime}\right]\left(\forall k^{\prime} \neq k\right)$. According to [26], the asymptotic rate approximation per time slot can be described as $R_{l}^{k^{\prime k}}(\mathrm{SNR})=d_{l}^{k} \log (\mathrm{SNR})+o(\log (\mathrm{SNR}))(k, l \in\{1,2\})$, where $\frac{o(\log (\mathrm{SNR}))}{\log (\mathrm{SNR})} \rightarrow 0$ as SNR $\rightarrow \infty$. Then, we define the sum DoF of the two-cluster MIMO mRIC with two users per cluster as

$$
d_{\text {sum }}=\sum_{l=1}^{2} \sum_{k=1}^{2} d_{l}^{k}=\sum_{l=1}^{2} \sum_{k=1, k^{\prime} \neq k}^{2} \lim _{\mathrm{SNR} \rightarrow \infty} \frac{R_{l}^{k^{k}}(\mathrm{SNR})}{\log (\mathrm{SNR})}
$$

where $d_{l}^{k}$ is the DoF of user $[l, k]$. The DoF of user $[l, k]$ can also be explained as the number of streams with decodability at user $\left[l, k^{\prime}\right][10]$. Therefore, user $[l, k]$ can obtain the DoF of $d_{l}^{k}$, if user $[l, k]$ transmits $d_{l}^{k}$ streams without interference and user $\left[l, k^{\prime}\right]$ can decode the $d_{l}^{k}$ desired streams.

\section{A DoF Upper Bound}

In this section, we provide a DoF upper bound of the considered MIMO mRIC based on cut-set bound. To get the DoF cut-set bound of the two-cluster mRIC, we can regard the two-cluster mRIC as a parallel connection of one-cluster two-way relay channels. Note that we assume $M_{1}^{1} \geq M_{1}^{2}$ and $M_{2}^{1} \geq M_{2}^{2}$. For conventional two-way relay channels, the DoF of cluster 1 is $d_{\text {cluster } 1} \leq \min \left(2 M_{1}^{2}, 2 N\right)$ by allowing user cooperation in cluster 1 and keeping users in cluster 2 silence. The DoF cut-set bound of the cluster 1 can be intuitively interpreted as follows. First, user [1,2] equipped with $M_{1}^{2}$ antennas can transmit and decode at most $M_{1}^{2}$ independent streams. Meanwhile, user [1,1] can decode at most $M_{1}^{2}$ independent desired streams. Thus, $d_{\text {cluster1 }}$ is upper bounded by $2 M_{1}^{2}$. Secondly, two relays have the total number of $2 N$ antennas and send at most $2 N$ independent streams to the users in cluster 1 . Thus, $d_{\text {cluster } 1}$ is also upper bounded by $2 N$. Similarly, if only the users in cluster 2 operate, the DoF of cluster 2 is given by $d_{\text {cluster } 2} \leq \min \left(2 M_{2}^{2}, 2 N\right)$. Adding up the DoF of two clusters, the DoF cut-set 
bound of the considered mRIC is given by

$$
d_{\text {sum }} \leq \min \left(2 M_{1}^{2}, 2 N\right)+\min \left(2 M_{2}^{2}, 2 N\right)
$$

If we have $N \geq M_{1}^{2}$ and $N \geq M_{2}^{2}$, the DoF cut-set bound becomes

$$
d_{\text {sum }} \leq 2 M_{1}^{2}+2 M_{2}^{2}
$$

If we consider a symmetric two-cluster MIMO mRIC with two users per cluster, the number of antennas at each user is equal to $\mathrm{M}$. Then, the DoF cut-set bound becomes

$$
d_{\text {sum }}=\left\{\begin{array}{l}
4 N, N<M \\
4 M, N \geq M
\end{array}\right.
$$

The DoF upper bounds in (7) and (8) will be used as benchmarks in the following transmission design.

\section{The Proposed Blind Interference Neutralization}

In this section, we provide a detailed transmission scheme to obtain the achievable DoF of the considered mRIC and verify that the DoF upper bound can be achieved. For convenience, we denote ( $\left.N \mid M_{1}^{1}, M_{1}^{2}, M_{2}^{1}, M_{2}^{2}\right)$ for the considered mRIC, where each relay has $N$ antennas and user $[l, k](l, k \in\{1,2\})$ is equipped with $M_{l}^{k}$ antennas.

\subsection{Motivating example}

We propose a blind interference neutralization scheme to neutralize ICI. The BIN scheme focuses on the design of the ICI reconstructing and neutralization matrices at two relays. As a motivating example, we consider a simple case with the system configuration $\left(N \mid M_{1}^{1}, M_{1}^{2}, M_{2}^{1}, M_{2}^{2}\right)=(9 \mid 5,4,5,4)$. For this case, the BIN scheme achieves the DoF of $d_{1}^{1}=d_{1}^{2}=d_{2}^{1}=d_{2}^{2}=4$. Therefore, the sum DoF of 16 can be obtained.

1) MAC phase

Using the method of antenna deactivation in [25], user [l,k] only uses $d_{l}^{k}$ antennas and the sizes of channel matrices $\mathbf{H}_{l}^{k, R_{i}}$ and $\mathbf{H}_{l}^{R_{i}, k}$ are $N \times d_{l}^{k}$ and $d_{l}^{k} \times N$, respectively. Since the BIN scheme only requires CSI at the relay, user $[l, k]$ directly sends $\mathbf{X}_{l}^{k}$ without precoding at the MAC phase.

2) The received signal at each relay

When all users simultaneously transmit signals to two relays, the received signal at relay $R_{i}$ can be denoted as

$$
\begin{aligned}
\mathbf{Y}^{R_{i}} & =\mathbf{H}_{1}^{R_{i}, 1} \mathbf{X}_{1}^{1}+\mathbf{H}_{1}^{R_{i}, 2} \mathbf{X}_{1}^{2}+\mathbf{H}_{2}^{R_{i}, 1} \mathbf{X}_{2}^{1}+\mathbf{H}_{2}^{R_{i}, 2} \mathbf{X}_{2}^{2}+\mathbf{Z}^{R_{i}}, i \in\{1,2\} \\
& =\sum_{l=1}^{2} \mathbf{F}_{l}^{R_{i}}\left(\begin{array}{c}
\mathbf{X}_{l}^{1} \\
\mathbf{X}_{l}^{2}
\end{array}\right)+\mathbf{Z}^{R_{i}}, i \in\{1,2\}
\end{aligned}
$$


where $\mathbf{F}_{l}^{R_{i}}=\left[\mathbf{H}_{l}^{R_{i}, 1} \mathbf{H}_{l}^{R_{i}, 2}\right]$ denotes the equivalent channel matrix with the size of $N \times\left(d_{l}^{1}+d_{l}^{2}\right)=9 \times 8 ; \mathbf{Z}^{R_{i}}$ denotes the $N \times 1$ AWGN vector at relay $R_{i}$. Note that all elements of channel matrices are drawn from a continuous distribution, $\mathbf{F}_{l}^{R_{i}}$ have full rank with probability 1.

The key of the BIN scheme is how to design the relay beamforming matrix $\mathbf{W}^{i}$ at relay $R_{i}$ to neutralize ICI. The relay beamforming matrix is designed to be composed of two components, i.e., the ICI reconstructing matrix $\mathbf{V}_{i}$ and the ICI neutralization matrix $\mathbf{U}_{i}$. The relay beamforming matrix can be written as :

$$
\mathbf{W}^{i}=\mathbf{U}_{i} \mathbf{V}_{i}^{H}=\sum_{n=1}^{L} \mathbf{u}_{i,(n)} \mathbf{v}_{i,(n)}^{H}, i \in\{1,2\}
$$

where $\mathbf{U}_{i}=\left[\mathbf{u}_{i,(1)}, \mathbf{u}_{i,(2)}, \ldots, \mathbf{u}_{i,(L)}\right], \mathbf{V}_{i}=\left[\mathbf{v}_{i,(1)}, \mathbf{v}_{i,(2)}, \ldots, \mathbf{v}_{i,(L)}\right], L=\max \left(d_{1}^{1}, d_{1}^{2}, d_{2}^{1}, d_{2}^{2}\right)=4$.

3) Design of the ICI reconstructing matrix at each relay

The goal of designing the ICI reconstructing matrix is to form a same linear combination of the signals transmitted from all users at two relays. In order to form a same linear combination, we design the ICI reconstructing matrix $\mathbf{V}_{i}(i \in\{1,2\})$ at relay $R_{i}$ so that the equivalent channels $\mathbf{V}_{i}^{H} \mathbf{F}_{1}^{R_{i}}$ observed by two relays $R_{1}$ and $R_{2}$ are identical. The conditions of the same linear combination can be written as

$$
\begin{aligned}
& \mathbf{v}_{1,(n)}^{H} \mathbf{F}_{1}^{R_{1}}=a(n) \mathbf{v}_{2,(n)}^{H} \mathbf{F}_{1}^{R_{2}} \\
& \mathbf{v}_{1,(n)}^{H} \mathbf{F}_{2}^{R_{1}}=b(n) \mathbf{v}_{2,(n)}^{H} \mathbf{F}_{2}^{R_{2}}
\end{aligned}
$$

where $a(n)$ and $b(n)(n \in\{1,2, \ldots L\})$ represent arbitrary non-zero real values. Here, we assume that the values $a(n)$ and $b(n)$ can be chosen to satisfy the conditions of $a(n) \neq b(n)$ and $\frac{a(1)}{b(1)} \neq \frac{a(2)}{b(2)} \neq \cdots \neq \frac{a(n)}{b(n)}$. If we choose $a(n)=b(n)$, we will eliminate both the ICI signal and the desired signal at the BC phase. We now explain why the conditions (11) and (12) can be satisfied. In order to insert (11) into (12), we should satisfy the invertibility of $\mathbf{F}_{1}^{R_{2}}$. We let relay $R_{2}$ use $d_{1}^{1}+d_{1}^{2}=8$ antennas and the size of $\mathbf{F}_{1}^{R_{2}}$ is equal to $8 \times 8$. Then, (11) can be rewritten as

$$
\frac{1}{a(n)} \mathbf{v}_{1,(n)}^{H} \mathbf{F}_{1}^{R_{1}} \mathbf{F}_{1}^{R_{2}^{-1}}=\mathbf{v}_{2,(n)}^{H}
$$

Combining (12) with (13), we further obtain

$$
\mathbf{v}_{1,(n)}^{H}\left(\mathbf{F}_{2}^{R_{1}}-\frac{b(n)}{a(n)} \mathbf{F}_{1}^{R_{1}} \mathbf{F}_{1}^{R_{2}^{-1}} \mathbf{F}_{2}^{R_{2}}\right)=\mathbf{0}
$$

Given $a(n)$ and $b(n)$ in (14), the ICI reconstructing vector $\mathbf{v}_{1,(n)}^{H}$ is determined by the left-hand 
nullspace of the matrix $\mathbf{F}_{2}^{R_{1}}-\frac{b(n)}{a(n)} \mathbf{F}_{1}^{R_{1}} \mathbf{F}_{1}^{R_{2}^{-1}} \mathbf{F}_{2}^{R_{2}}$. Since the size of the matrix $\mathbf{F}_{2}^{R_{1}}-\frac{b(n)}{a(n)} \mathbf{F}_{1}^{R_{1}} \mathbf{F}_{1}^{R_{2}^{-1}} \mathbf{F}_{2}^{R_{2}}$ is $N \times\left(d_{2}^{1}+d_{2}^{2}\right)=9 \times 8$, null $\left(\left(\mathbf{F}_{2}^{R_{1}}-\frac{b(n)}{a(n)} \mathbf{F}_{1}^{R_{1}} \mathbf{F}_{1}^{R_{2}^{-1}} \mathbf{F}_{2}^{R_{2}}\right)^{T}\right)=1$ and $\mathbf{v}_{1,(n)}$ exists. Since we assume $\frac{a(1)}{b(1)} \neq \frac{a(2)}{b(2)} \neq \cdots \neq \frac{a(n)}{b(n)}, \mathbf{v}_{1,(1)}, \mathbf{v}_{1,(2)}, \ldots, \mathbf{v}_{1,(L)}$ are linearly independent with probability 1 . After obtaining $\mathbf{v}_{1,(n)}$, the ICI reconstructing vector $\mathbf{v}_{2,(n)}$ can be achieved from (13). Therefore, the conditions (11) and (12) hold.

Based on (11) and (12), the received signal at relay $R_{i}$ becomes

$$
\begin{gathered}
\mathbf{v}_{1,(n)}^{H} \mathbf{Y}^{R_{1}}=L^{(n)}\left(\mathbf{X}_{1}^{1}, \mathbf{X}_{1}^{2}\right)+L^{(n)}\left(\mathbf{X}_{2}^{1}, \mathbf{X}_{2}^{2}\right)+\mathbf{v}_{1,(n)}^{H} \mathbf{Z}^{R_{1}} \\
\mathbf{v}_{2,(n)}^{H} \mathbf{Y}^{R_{2}}=\frac{1}{a(n)} L^{(n)}\left(\mathbf{X}_{1}^{1}, \mathbf{X}_{1}^{2}\right)+\frac{1}{b(n)} L^{(n)}\left(\mathbf{X}_{2}^{1}, \mathbf{X}_{2}^{2}\right)+\mathbf{v}_{2,(n)}^{H} \mathbf{Z}^{R_{2}}
\end{gathered}
$$

where $\quad L^{(n)}\left(\mathbf{X}_{1}^{1}, \mathbf{X}_{1}^{2}\right)=\mathbf{v}_{1,(n)}^{H} \mathbf{H}_{1}^{R_{1}, 1} \mathbf{X}_{1}^{1}+\mathbf{v}_{1,(n)}^{H} \mathbf{H}_{1}^{R_{1}, 2} \mathbf{X}_{1}^{2} \quad$ and $\quad L^{(n)}\left(\mathbf{X}_{2}^{1}, \mathbf{X}_{2}^{2}\right)=\mathbf{v}_{1,(n)}^{H} \mathbf{H}_{2}^{R_{1}, 1} \mathbf{X}_{2}^{1}+\mathbf{v}_{1,(n)}^{H} \mathbf{H}_{2}^{R_{1}, 2} \mathbf{X}_{2}^{2}$ denote the linear combination of the signals transmitted from user $[1, k]$ and user $[2, k]$, $k \in\{1,2\}$, respectively. According to the design of the ICI reconstructing matrix, relay $R_{1}$ and $R_{2}$ have the same linear combinations $L^{(n)}\left(\mathbf{X}_{1}^{1}, \mathbf{X}_{1}^{2}\right)$ and $L^{(n)}\left(\mathbf{X}_{2}^{1}, \mathbf{X}_{2}^{2}\right)$.

4) Design of the ICI neutralization matrix at each relay

We design the ICI neutralization matrix $\mathbf{U}_{i}$ to neutralize ICI at the BC phase. During the BC phase, two relays cooperatively transmit the reconstructed signals $\mathbf{v}_{1,(n)}^{H} \mathbf{Y}^{R_{1}}$ and $\mathbf{v}_{2,(n)}^{H} \mathbf{Y}^{R_{2}}$ to all users along with the ICI neutralization vectors $\mathbf{u}_{1,(n)}$ and $\mathbf{u}_{2,(n)}$, respectively. In order to exchange messages without ICI, we need to neutralize $L^{(n)}\left(\mathbf{X}_{2}^{1}, \mathbf{X}_{2}^{2}\right)$ at user $[1, k]$ and $L^{(n)}\left(\mathbf{X}_{1}^{1}, \mathbf{X}_{1}^{2}\right)$ at user [2,k] $(k \in\{1,2\})$, respectively. The neutralization conditions are denoted as

$$
\begin{gathered}
-b(n)\left[\begin{array}{l}
\mathbf{H}_{1}^{1, R_{1}} \\
\mathbf{H}_{1}^{2, R_{1}}
\end{array}\right] \mathbf{u}_{1,(n)}=\left[\begin{array}{l}
\mathbf{H}_{1}^{1, R_{2}} \\
\mathbf{H}_{1}^{2, R_{2}}
\end{array}\right] \mathbf{u}_{2,(n)} \\
-a(n)\left[\begin{array}{l}
\mathbf{H}_{2}^{1, R_{1}} \\
\mathbf{H}_{2}^{2, R_{1}}
\end{array}\right] \mathbf{u}_{1,(n)}=\left[\begin{array}{l}
\mathbf{H}_{2}^{1, R_{2}} \\
\mathbf{H}_{2}^{2, R_{2}}
\end{array}\right] \mathbf{u}_{2,(n)}
\end{gathered}
$$

We now explain why the conditions (17) and (18) can be satisfied. If we denote $\mathbf{G}_{l}^{R_{i}}=\left[\left(\mathbf{H}_{l}^{1, R_{i}}\right)^{H}\left(\mathbf{H}_{l}^{2, R_{i}}\right)^{H}\right]^{H}$, the size of $\mathbf{G}_{1}^{R_{2}}$ is also $8 \times 8$. Then, (17) and (18) can be rewritten as

$$
-b(n) \mathbf{G}_{1}^{R_{1}^{-1}} \mathbf{G}_{1}^{R_{1}} \mathbf{u}_{1,(n)}=\mathbf{u}_{2,(n)}
$$




$$
\mathbf{G}_{2}^{R_{1}} \mathbf{u}_{1,(n)}=-\frac{1}{a(n)} \mathbf{G}_{2}^{R_{2}} \mathbf{u}_{2,(n)}
$$

Combining (19) with (20), we further obtain

$$
\left(\mathbf{G}_{2}^{R_{1}}-\frac{b(n)}{a(n)} \mathbf{G}_{2}^{R_{2}} \mathbf{G}_{1}^{R_{2}^{-1}} \mathbf{G}_{1}^{R_{1}}\right) \mathbf{u}_{1,(n)}=\mathbf{0}
$$

The ICI neutralization vector $\mathbf{u}_{1,(n)}$ can be calculated as the nulling vector of the matrix $\mathbf{G}_{2}^{R_{1}}-\frac{b(n)}{a(n)} \mathbf{G}_{2}^{R_{2}} \mathbf{G}_{1}^{R^{-1}} \mathbf{G}_{1}^{R_{1}}$ with the size of $8 \times 9$. Since null $\left(\mathbf{G}_{2}^{R_{1}}-\frac{b(n)}{a(n)} \mathbf{G}_{2}^{R_{2}} \mathbf{G}_{1}^{R_{2}^{-1}} \mathbf{G}_{1}^{R_{1}}\right)=1, \mathbf{u}_{1,(n)}$ exists. In a similar manner as the MAC phase, $\mathbf{u}_{1,(1)}, \mathbf{u}_{1,(2)}, \cdots, \mathbf{u}_{1,(L)}$ are linearly independent with probability 1 . Then, we can decide the ICI neutralization vector $\mathbf{u}_{2,(n)}$ from (19). Therefore, the conditions (17) and (18) hold.

5) BC phase

Two relays broadcast their received signals $\mathbf{Y}^{R_{1}}$ and $\mathbf{Y}^{R_{2}}$ by using the relay beamforming matrices $\mathbf{W}^{1}=\sum_{n=1}^{L} \mathbf{u}_{1,(n)} \mathbf{v}_{1,(n)}^{H}=\mathbf{U}_{1} \mathbf{V}_{1}^{H}$ and $\mathbf{W}^{2}=\sum_{n=1}^{L} \mathbf{u}_{2,(n)} \mathbf{v}_{2,(n)}^{H}=\mathbf{U}_{2} \mathbf{V}_{2}^{H}$, respectively. Then, the received signal at user $[1,1]$ can be obtained as

$$
\begin{aligned}
\mathbf{Y}_{1}^{1} & =\mathbf{H}_{1}^{1, R_{1}} \mathbf{W}^{1} \mathbf{Y}^{R_{1}}+\mathbf{H}_{1}^{1, R_{2}} \mathbf{W}^{2} \mathbf{Y}^{R_{2}}+\mathbf{N}_{1}^{1} \\
& =\mathbf{H}_{1}^{1, R_{1}}\left(\sum_{n=1}^{L} \mathbf{u}_{1,(n)} \mathbf{v}_{1,(n)}^{H}\right) \mathbf{Y}^{R_{1}}+\mathbf{H}_{1}^{1, R_{2}}\left(\sum_{n=1}^{L} \mathbf{u}_{2,(n)} \mathbf{v}_{2,(n)}^{H}\right) \mathbf{Y}^{R_{2}}+\mathbf{N}_{1}^{1} \\
& =\left(\sum_{n=1}^{L} \mathbf{H}_{1}^{1, R_{1}} \mathbf{u}_{1,(n)} \mathbf{v}_{1,(n)}^{H}\right) \mathbf{Y}^{R_{1}}-\left(\sum_{n=1}^{L} b(n) \mathbf{H}_{1}^{1, R_{1}} \mathbf{u}_{1,(n)} \mathbf{v}_{2,(n)}^{H}\right) \mathbf{Y}^{R_{2}}+\mathbf{N}_{1}^{1} \\
& =\mathbf{H}_{1}^{1, R_{1}} \sum_{n=1}^{L} \mathbf{u}_{1,(n)}\left(\mathbf{v}_{1,(n)}^{H} \mathbf{Y}^{R_{1}}-b(n) \mathbf{v}_{2,(n)}^{H} \mathbf{Y}^{R_{2}}\right)+\mathbf{N}_{1}^{1} \\
& =\mathbf{H}_{1}^{1, R_{1}} \sum_{n=1}^{L} \mathbf{u}_{1,(n)}\left(L^{(n)}\left(\mathbf{X}_{1}^{1}, \mathbf{X}_{1}^{2}\right)-\frac{b(n)}{a(n)} L^{(n)}\left(\mathbf{X}_{1}^{1}, \mathbf{X}_{1}^{2}\right)\right)+\tilde{\mathbf{N}}_{1}^{1}
\end{aligned}
$$

where $\tilde{\mathbf{N}}_{1}^{1}=\mathbf{H}_{1}^{1, R_{1}} \mathbf{W}^{1} \mathbf{Z}^{R_{1}}+\mathbf{H}_{1}^{1, R_{2}} \mathbf{W}^{2} \mathbf{Z}^{R_{2}}+\mathbf{N}_{1}^{1}$. The equation (22) shows that the ICI $L^{(n)}\left(\mathbf{X}_{2}^{1}, \mathbf{X}_{2}^{2}\right)$ is neutralized at user [1,1]. By using the zero forcing matrix $\mathbf{D}_{1}^{1}$ to remove self-interference, user $[1,1]$ can decode its desired signal $\mathbf{X}_{1}^{2}$ as:

$$
\begin{aligned}
\hat{\mathbf{Y}}_{1}^{1} & =\mathbf{D}_{1}^{1} \mathbf{H}_{1}^{1, R_{1}} \sum_{n=1}^{L} \mathbf{u}_{1,(n)}\left(L^{(n)}\left(\mathbf{X}_{1}^{1}, \mathbf{X}_{1}^{2}\right)-\frac{b(n)}{a(n)} L^{(n)}\left(\mathbf{X}_{1}^{1}, \mathbf{X}_{1}^{2}\right)\right)+\tilde{\mathbf{N}}_{1}^{1} \\
& =\mathbf{D}_{1}^{1} \mathbf{H}_{1}^{1, R_{1}} \sum_{n=1}^{L} \mathbf{u}_{1,(n)}\left(\frac{a(n)-b(n)}{a(n)}\right) L^{(n)}\left(\mathbf{X}_{1}^{1}, \mathbf{X}_{1}^{2}\right)+\tilde{\mathbf{N}}_{1}^{1} \\
& =\mathbf{D}_{1}^{1} \mathbf{H}_{1}^{1, R_{1}} \mathbf{U}_{1} \mathbf{\Sigma}_{1} \mathbf{V}_{1}^{H} \mathbf{H}_{1}^{R_{1}, 2} \mathbf{X}_{1}^{2}+\tilde{\mathbf{N}}_{1}^{1}
\end{aligned}
$$


where $\boldsymbol{\Sigma}_{1}=\operatorname{diag}\left(\frac{a(1)-b(1)}{a(1)}, \frac{a(2)-b(2)}{a(2)}, \cdots, \frac{a(L)-b(L)}{a(L)}\right)$ is the $L \times L$ diagonal matrix whose the $n$-th element is defined as $\frac{a(n)-b(n)}{a(n)} ; \mathbf{D}_{1}^{1}$ is the $d_{l}^{2} \times d_{l}^{1}$ zero forcing matrix. Since the ICI reconstructing and neutralization matrices $\mathbf{V}_{1}$ and $\mathbf{U}_{1}$ have been designed to be full rank, the rank of the effective channel $\mathbf{D}_{1}^{1} \mathbf{H}_{1}^{1, R_{1}} \mathbf{U}_{1} \boldsymbol{\Sigma}_{1} \mathbf{V}_{1}^{H} \mathbf{H}_{1}^{R_{1}, 2}$ is guaranteed to be $d_{1}^{2}=4$. Therefore, user $[1,1]$ can decode $d_{1}^{2}=4$ desired streams.

In a similar way, user [1,2], user [2,1] and user [2,2] can decode $d_{1}^{1}, d_{2}^{2}$ and $d_{2}^{1}$ data streams, respectively. As a result, user [1,1] and user [1,2] achieve the DoF of $d_{1}^{1}+d_{1}^{2}=8$, and user $[2,1]$ and user [2,2] achieve the DoF of $d_{2}^{1}+d_{2}^{2}=8$. Therefore, the sum DoF of 16 can be achieved.

\subsection{Extension to the general system configuration}

Based on the above analysis, the neutralization conditions (17) and (18) can be satisfied if (11) and (14) hold. According to (11) and (14), the feasibility conditions of the BIN scheme can be written as $d_{1}^{1}+d_{1}^{2}=N$ and $d_{2}^{1}+d_{2}^{2} \leq N-1$. Note that $N$ is not the entire number of antennas but a subset of antennas at relay $R_{i}$. The following theorem establishes the achievable DoF of the considered mRIC with the general system configuration $\left(N \mid M_{1}^{1}, M_{1}^{2}, M_{2}^{1}, M_{2}^{2}\right)$.

Theorem 1: For the two-cluster mRIC with two users per cluster, we can show that the following DoF is achievable:

$$
\operatorname{DoF}= \begin{cases}2 N-1, \quad N \leq 2 M_{2}^{2} \\ N+2 M_{2}^{2}, \quad 2 M_{2}^{2}<N \leq 2 M_{1}^{2} \\ 2 M_{1}^{2}+2 M_{2}^{2}, \quad N>2 M_{1}^{2}\end{cases}
$$

Proof: To transmit data streams without ICI, we need to satisfy the feasibility conditions of the BIN scheme $d_{1}^{1}+d_{1}^{2}=N$ and $d_{2}^{1}+d_{2}^{2} \leq N-1$. When $N \leq 2 M_{2}^{2}$, the DoF of $2 N-1$ can directly be achieved by selecting $d_{1}^{1}+d_{1}^{2}=N$ and $d_{2}^{1}+d_{2}^{2}=N-1$. When $2 M_{2}^{2}<N \leq 2 M_{1}^{2}$, both user [2,1] and user [2,2] can transmit $M_{2}^{2}$ streams to satisfy the condition of $d_{2}^{1}+d_{2}^{2} \leq N-1$. Since $N \leq 2 M_{1}^{2}$, users in cluster 1 can use a subset of antennas to satisfy the condition of $d_{1}^{1}+d_{1}^{2}=N$. Therefore, the DoF of $N+2 M_{2}^{2}$ can be achieved in the case of $2 M_{2}^{2}<N \leq 2 M_{1}^{2}$. Moreover, the result shows that the DoF of cluster 2 is optimal in this scenario. When $N>2 M_{1}^{2}$, two relays $R_{1}$ and $R_{2}$ only use $2 M_{2}^{2}+1$ and $2 M_{1}^{2}$ antennas, respectively. The feasibility conditions can be satisfied by selecting $d_{1}^{1}=d_{1}^{2}=M_{1}^{2}$ and $d_{2}^{1}=d_{2}^{2}=M_{2}^{2}$. Therefore, the DoF upper bound $2 M_{1}^{2}+2 M_{2}^{2}$ can be obtained in the case of $N>2 M_{1}^{2}$. Finally, we summarize the proposed BIN scheme in Table 2. 
Table 2. The procedure of BIN scheme for the considered mRIC

1: The equivalent channel matrix $\mathbf{F}_{l}^{R_{i}}$ is constructed by utilizing the uplink channel matrix $\mathbf{H}_{l}^{R_{i}, k}$ $(i, l, k \in\{1,2\})$ according to (9).

2: Two relays jointly design the interference reconstructing matrix $\mathbf{V}_{i}(i \in\{1,2\})$ to obtain the same linear combination of $L^{(n)}\left(\mathbf{X}_{l}^{1}, \mathbf{X}_{l}^{2}\right)$ according to (11) and (12).

3: The equivalent channel matrix $\mathbf{G}_{l}^{R_{i}}$ is constructed by utilizing the downlink channel matrix $\mathbf{H}_{l}^{k, R_{i}} \quad(i, l, k \in\{1,2\})$ according to (17) and (18).

4: Two relays jointly design the interference neutralization matrix $\mathbf{U}_{i}(i \in\{1,2\})$ to eliminate ICI according to (19) and (20).

Remark: Different from the scheme in [16, 19], the beamforming matrix design of the proposed BIN scheme is completed only at two relays. Therefore, users can be blind. Studying the DoF of the considered mRIC can provide a heuristic thought, which the overheard information of multiple relays can be utilized to neutralize ICI. Furthermore, asymmetry in the number of antennas at the users can be utilized to facilitate the BIN scheme. For example, as long as the condition of $N>2 M_{2}^{2}$ is satisfied, user [2,1] can randomly select $M_{2}^{2}$ antennas to satisfy the condition of $d_{2}^{1}+d_{2}^{2}=N-1$. Therefore, the feasibility condition of the BIN scheme is extended.

\section{Numerical Results}

In this section, we provide numerical results to evaluate the sum rate performance of the BIN scheme. We demonstrate that the BIN scheme exactly attains the achievable DoF derived in Section 4. To analyze the performance gain, the BIN scheme is compared with the conventional TDMA scheme, the asymmetric SSA scheme in [13] and the symmetric SSA scheme in [14] and [15]. The channel is modeled as i.i.d. complex Gaussian distribution with zero mean and unit variance. The numerical results are averaged over 1000 independent Monte Carlo runs. Note that we denote $\left(N \mid M_{1}^{1}, M_{1}^{2}, M_{2}^{1}, M_{2}^{2}\right)$ for the considered mRIC where each relay has $N$ antennas and user $[l, k](l, k \in\{1,2\})$ is equipped with $M_{l}^{k}$ antennas.

We first explain how to compute the sum rate of the considered mRIC by applying the BIN scheme. Note that $R_{1}^{12}$ denotes the sum rate from user [1,2] to user [1,1]. We calculate $R_{1}^{12}$ as a representative. According to (23), the received signal at user $[1,1]$ can be described as

$$
\hat{\mathbf{Y}}_{1}^{1}=\tilde{\mathbf{H}}_{1}^{1, R_{1}} \mathbf{X}_{1}^{2}+\tilde{\mathbf{N}}_{1}^{1}
$$

where $\tilde{\mathbf{H}}_{1}^{1, R_{1}}=\mathbf{D}_{1}^{1} \mathbf{H}_{1}^{1, R_{1}} \mathbf{U}_{1} \boldsymbol{\Sigma}_{1} \mathbf{V}_{1}^{H} \mathbf{H}_{1}^{R_{1}, 2}$ and $\tilde{\mathbf{N}}_{1}^{1}$ represent the equivalent channel matrix and noise vector, respectively. According to [27], we can calculate the sum rate $R_{1}^{12}$ in bits per channel use by (26)

$$
R_{1}^{12}=\log _{2}\left[\operatorname{det}\left(\mathbf{I}+\tilde{\mathbf{H}}_{1}^{1, R_{1}} \varepsilon\left(\mathbf{X}_{1}^{2}\left(\mathbf{X}_{1}^{2}\right)^{H}\right)\left(\tilde{\mathbf{H}}_{1}^{1, R_{1}}\right)^{H} \varepsilon\left(\tilde{\mathbf{N}}_{1}^{1}\left(\tilde{\mathbf{N}}_{1}^{1}\right)^{H}\right)^{-1}\right)\right]
$$

where $\operatorname{det}[\cdot]$ and $\varepsilon[\cdot]$ denote the determinant and expectation operation, respectively. The sum rate $R_{1}^{21}, R_{2}^{12}$ and $R_{2}^{21}$ can be computed in a similar way. The total sum rate is calculated by 
(27).

$$
R_{\text {total }}=R_{1}^{12}+R_{1}^{21}+R_{2}^{12}+R_{2}^{21}
$$

Fig. 2 illustrates the sum rate performance of the BIN scheme as a function of SNR according to different system configurations. The sum rate of the considered mRIC is calculated by (27). Three color lines are drawn based on three different cases of Theorem 1 , i.e., case 1: $N \leq 2 M_{2}^{2}$; case 2: $2 M_{2}^{2}<N \leq 2 M_{1}^{2}$; case $3: N>2 M_{1}^{2}$. In each case, the comparison of two different system configurations with the same total number of antennas is provided. The system configuration of each color line is shown as follows: blue line (case 1): $(5 \mid 6,3,3,3)$ and $(6 \mid 5,3,3,3)$; green line (case 2$):(7 \mid 6,6,3,3)$ and $(8 \mid 5,4,5,4)$; red line (case 3$):(11 \mid 8,5,8,5)$ and $(13 \mid 6,6,6,6)$. The slopes of the sum rate curves at high SNR are equal to $\frac{\mathrm{DoF}}{10 \lg 2}$ according to the Shannon capacity formula. We can observe that the slopes of the blue, green and red lines match with the theoretical DoF of $2 N-1, N+2 M_{2}^{2}$ and $2 M_{1}^{2}+2 M_{2}^{2}$ well at the high SNR regime, respectively. Moreover, we can see that the lines with the same total number of antennas can obtain different DoF. The reason is that increasing the number of antennas at the following nodes can improve the DoF gain: 1) relay for case 1,2) relay and user [2,2] for case 2,3 ) user $[1,2]$ and user $[2,2]$ for case 3 . The result indicates that the BIN scheme is feasible and asymmetry in the number of antennas at users can be utilized to facilitate the BIN scheme.

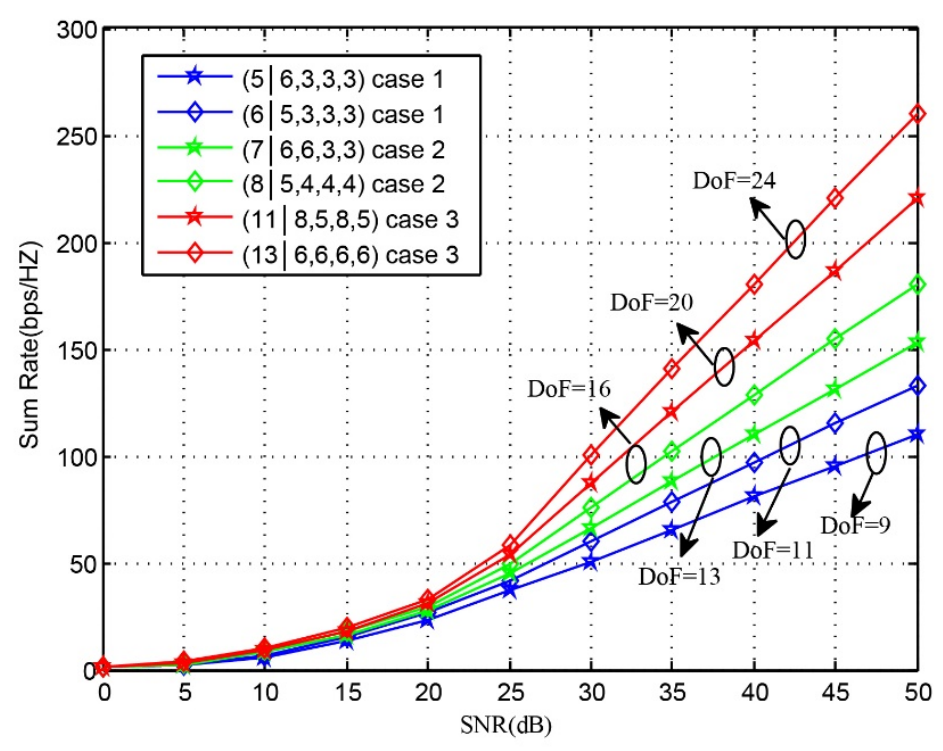

Fig. 2. The sum rate for the considered mRIC with various antenna configurations

To show the superiority of the BIN scheme, we first consider a baseline approach, i.e., the conventional TDMA-NC scheme in [14]. The TDMA-NC scheme requires two time slots to transmit streams without ICI. During the first time slot, relay $R_{1}$ and users in cluster 1 transmit 
data streams, while relay $R_{2}$ and users in cluster 2 keep silence. During the second time slot, relay $R_{2}$ and users in cluster 2 transmit data streams, while relay $R_{1}$ and users in cluster 1 keep silence. Therefore, the DoF of $M_{1}^{2}+M_{2}^{2}$ can be trivially obtained in case of $N>M_{1}^{2}$ by using the TDMA-NC scheme. Fig. 3 shows the sum rate versus SNR performance result, which compares the BIN scheme with the TDMA-NC scheme under three system configurations. It is illustrated that the BIN scheme provides about 28.5\%, 85.7\% and 100\% DoF improvement compared to the TDMA-NC scheme under the following system configurations: $(5 \mid 5,4,5,3)$, $(6 \mid 6,3,3,3)$ and $(9 \mid 5,4,5,4)$. The main reason is that the TDMA-NC scheme uses multiple time slots to ensure the transmission without ICI, whereas the BIN scheme can send streams without ICI at a single time slot. Moreover, we show that the BIN scheme can provide at most $100 \%$ DoF gain over the TDMA-NC scheme. This is because the DoF upper bound of the two-cluster mRIC is $2 M_{1}^{2}+2 M_{2}^{2}$.

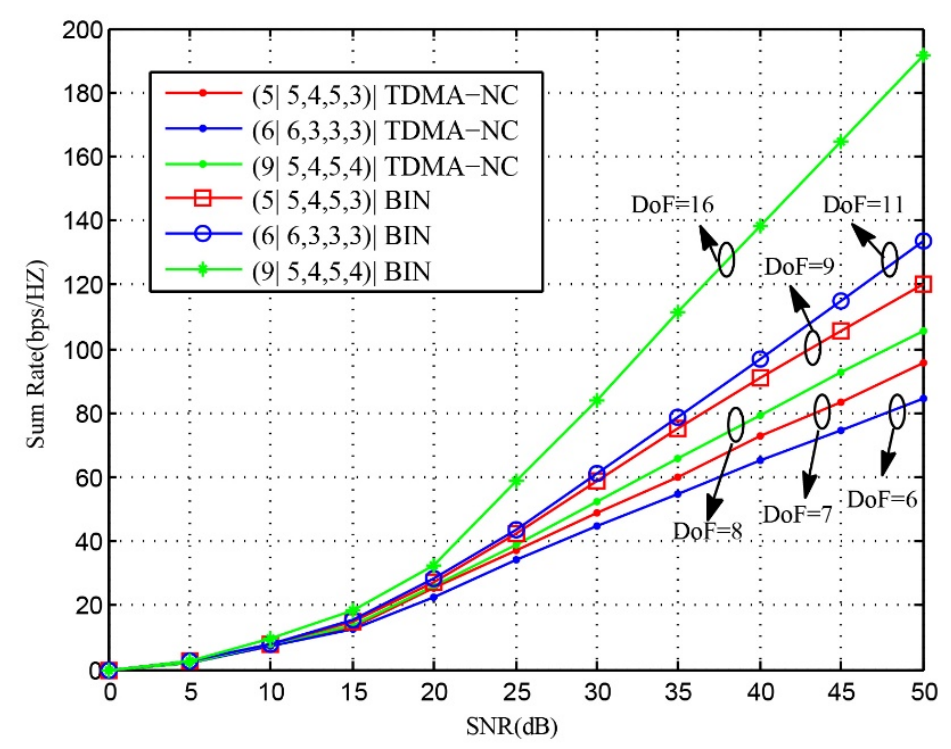

Fig. 3. Comparison of sum rate performance between the BIN scheme and the TDMA-NC scheme under the same system configuration

In Fig. 4, the sum rate comparison of the asymmetric SSA scheme (ASSA) in [13] and the BIN scheme is given. We focus on the case where the ASSA scheme cannot obtain the optimal achievable DoF in the considered mRIC. This is because the optimal achievable DoF cannot be obtained in most cases by using the ASSA scheme. In other words, the optimal achievable DoF can be obtained only if the number of antennas at users satisfies some specific conditions. The comparison is accomplished under three system configurations, which are three different cases of Theorem 1. In particular, case $1:(5 \mid 4,3,4,3)$; case 2 : $(8 \mid 7,5,6,3)$; case 3 : $(11 \mid 10,5,8,5)$. It is illustrated that the BIN scheme provides about $12.5 \%, 40 \%$ and $66.7 \%$ DoF improvement compared to the SSA scheme in case 1, case 2 and case 3, respectively. This is due to the fact that antennas at the relay cannot be fully utilized to construct the intersection 
space by using the ASSA scheme. For example, when the system configuration is $(11 \mid 10,5,8,5)$, all users can only construct a 6 -dimensional intersection space at the relay, which implies that there is a 5-dimensional redundant space at the relay. On the contrary, the BIN scheme can effectively utilize the 20-dimensional space at two relays to obtain a higher DoF than the ASSA scheme. The result indicates that the BIN is more effective than the ASSA scheme in the considered scenario.

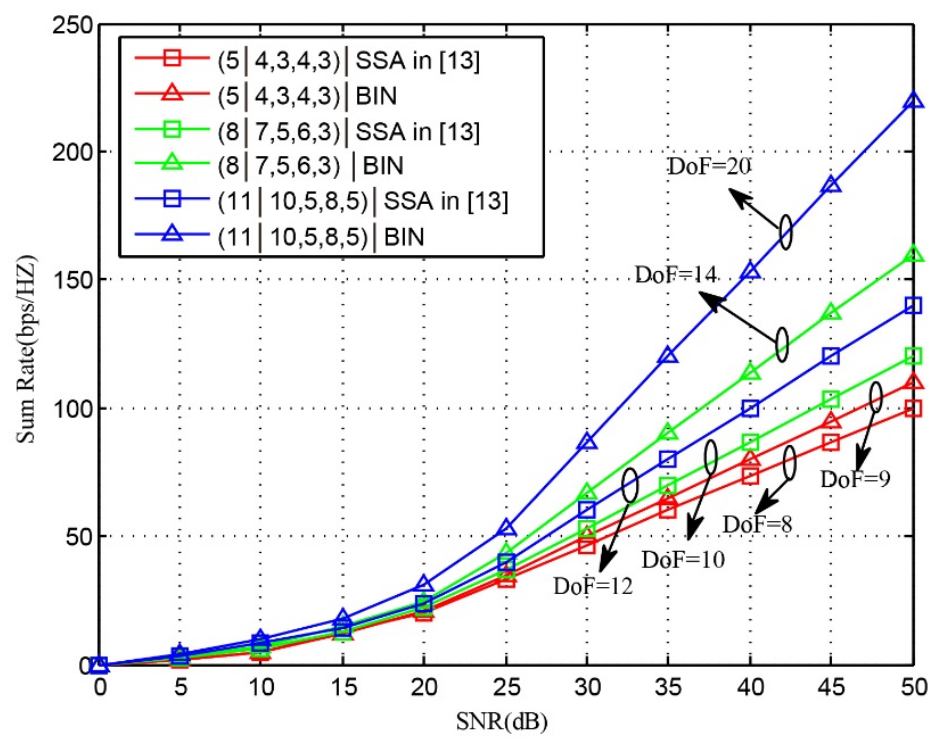

Fig. 4. Sum rate comparison between the BIN scheme and the SSA scheme in [13]

In Fig. 5, we plot the achievable DoF achieved by different schemes against the number of antennas at the relay $N$. In order to compare the BIN scheme with the symmetric SSA in [14] and [15], we assume that all users have the same number of antennas, i.e., $M_{l}^{k}=M$. For comparison, we also consider the DoF upper bound obtained in Section 3. The red line represents the DoF upper bound. The yellow, blue and green lines represent the achievable DoF derived in [14], [15] and this paper, respectively. We can observe that the achievable DoF of $2 N$ can only be obtained in the range of $N \in\left(0, \frac{4}{3} M\right]$ by using the conventional SSA scheme in [14]. This is because the $(4 M-2 N)$-dimensional intersection space at the relay is strictly less than $N$ in the range of $N \in\left(\frac{4}{3} M, \infty\right)$ so that there are antenna redundancies at the relay. It is further illustrated that the DoF upper bound can be obtained in the range of $N \in[3 M, \infty)$ by using the SSA scheme in [15]. However, we can see that the achievable DoF of the BIN scheme coincides with the DoF upper bound in the range of $N \in[2 M+1, \infty)$, which implies that the BIN scheme can achieve the DoF upper bound in a wider range than the SSA scheme in [15]. Moreover, we observe that the blue line and the green line have a crossover point at $N=\frac{4}{3} M+1$, which implies that the BIN scheme outperforms the SSA scheme in [15] 
in the range of $N \in\left[\frac{4}{3} M+1,3 M\right]$. The reason for the increase of DoF comes from that the BIN scheme can utilize the overheard information of two relays whereas the SSA scheme can only use the signal space at one relay node.

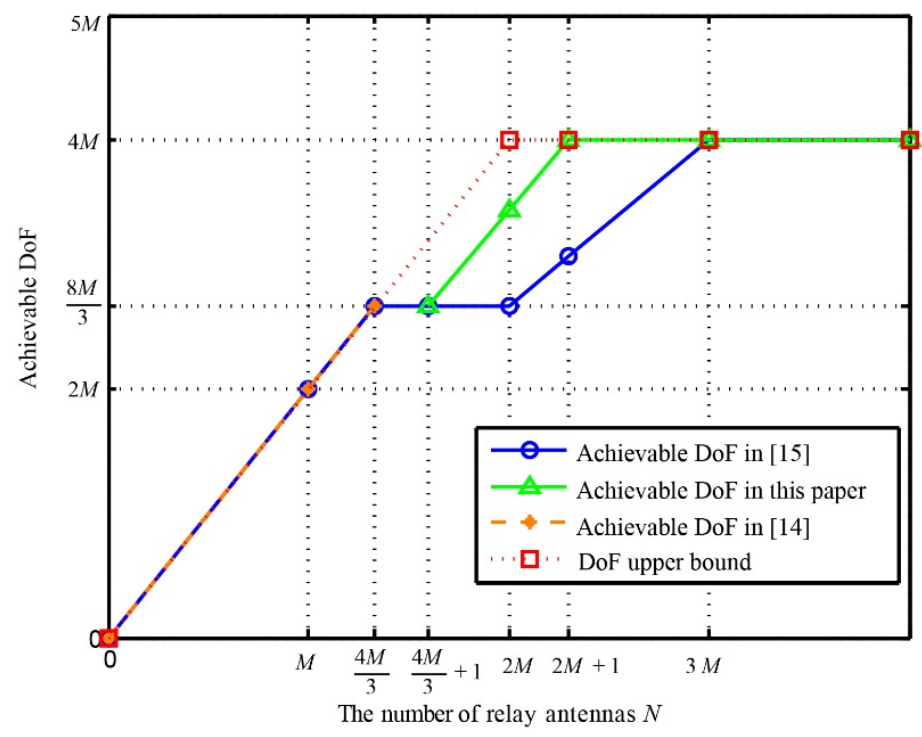

Fig. 5. Achievable DoF obtained by different schemes for the symmetric two-cluster mRIC with two users per cluster

\section{Conclusion}

In this paper, we investigated the DoF of the two-cluster MIMO mRIC with two relays and two users per cluster, where users within the same cluster exchange messages among themselves with the help of two relays. The DoF upper bound of the considered MIMO mRIC was derived based on cut-set bound. To approach the DoF upper bound, the BIN scheme was proposed to jointly design beamforming matrices at two relays to eliminate the inter-cluster interference. By using the BIN scheme, the DoF upper bound can be achieved without CSI at users. Through theoretical analysis and numerical simulations, we showed that the DoF upper bound $2 M_{1}^{2}+2 M_{2}^{2}$ can be obtained in the case of $N>2 M_{1}^{2}$. From simulation results, we showed that the BIN scheme can provide significant performance gain over the conventional TDMA-NC scheme in terms of DoF. Furthermore, we showed that the BIN scheme is a superior approach to the existing SSA schemes for the considered mRIC. It is an interesting future topic to study the extension of our work into a general mRIC, where there are arbitrary number of clusters and arbitrary number of users per cluster. 


\section{References}

[1] B. Rankov and A. Wittneben, "Spectral efficient protocols for half-duplex fading relay channels," Selected Areas in Communications, IEEE Journal on, vol. 25, no. 2, pp. 379-389, 2007. Article (CrossRef Link)

[2] W. Nam, S.-Y. Chung and Y.H. Lee, "Capacity of the Gaussian two-way relay channel to within 1/2 bit,” Information Theory, IEEE Transactions on, vol. 56, no. 11, pp. 5488-5494, 2010. Article (CrossRef Link)

[3] A.S. Avestimehr, A. Sezgin and D.N. Tse, "Capacity of the two-way relay channel within a constant gap,” European Transactions on Telecommunications, vol. 21, no. 4, pp. 363-374, 2010. Article (CrossRef Link)

[4] S. Zhang and S.-C. Liew, "Channel coding and decoding in a relay system operated with physical-layer network coding," Selected Areas in Communications, IEEE Journal on, vol. 27, no. 5, pp. 788-796, 2009. Article (CrossRef Link)

[5] M.P. Wilson, K. Narayanan, H.D. Pfister and A. Sprintson, “Joint physical layer coding and network coding for bidirectional relaying,” Information Theory, IEEE Transactions on, vol. 56, no. 11, pp. 5641-5654, 2010. Article (CrossRef Link)

[6] P. Popovski and H. Yomo, "Physical network coding in two-way wireless relay channels," in Proc. of Communications, ICC'07, IEEE International Conference on, IEEE, pp. 707-712, 2007. Article (CrossRef Link)

[7] D. Gunduz, A. Yener, A. Goldsmith and H.V. Poor, “The multiway relay channel," Information Theory, IEEE Transactions on, vol. 59, no. 1, pp. 51-63, 2013. Article (CrossRef Link)

[8] S.A. Jafar and S. Shamai, “Degrees of freedom region of the MIMO X channel,” Information Theory, IEEE Transactions on, vol. 54, no. 1, pp. 151-170, 2008. Article (CrossRef Link)

[9] V.R. Cadambe and S.A. Jafar, "Degrees of freedom of wireless X networks," in Proc. of Information Theory, ISIT 2008, IEEE International Symposium on, IEEE, pp. 1268-1272, 2008. Article (CrossRef Link)

[10] V.R. Cadambe and S.A. Jafar, "Interference alignment and degrees of freedom of the K-user interference channel,” Information Theory, IEEE Transactions on, vol. 54, no. 8, pp. 3425-3441, 2008. Article (CrossRef Link)

[11] N. Lee, J.-B. Lim and J. Chun, "Degrees of freedom of the MIMO Y channel: Signal space alignment for network coding," Information Theory, IEEE Transactions on, vol. 56, no. 7, pp. 3332-3342, 2010. Article (CrossRef Link)

[12] K. Lee, N. Lee and I. Lee, “Achievable degrees of freedom on K-user Y channels," Wireless Communications, IEEE Transactions on, vol. 11, no. 3, pp. 1210-1219, 2012. Article (CrossRef Link)

[13] Y. Tian and A. Yener, "Degrees of freedom optimal transmission for the two-cluster MIMO multi-way relay channel,” in Proc. of Communications (ICC), 2013 IEEE International Conference on, IEEE, pp. 3344-3348, 2013. Article (CrossRef Link)

[14] Y. Tian and A. Yener, "Signal space alignment and degrees of freedom for the two-cluster multi-way relay channel," in Proc. of Communications in China (ICCC), 2012 1st IEEE International Conference on, IEEE, pp. 12-17, 2012. Article (CrossRef Link)

[15] R. Wang, X. Yuan and M. Tao, "Degrees of freedom of MIMO Multiway relay channel with clustered pairwise exchange,” Selected Areas in Communications IEEE Journal on, vol. 33, pp. 337-351, 2015. Article (CrossRef Link)

[16] T. Gou, S.A. Jafar, C. Wang, S.-W. Jeon and S.-Y. Chung, “Aligned interference neutralization and the degrees of freedom of the $2 \times 2 \times 2$ interference channel," Information Theory, IEEE Transactions on, vol. 58, no. 7, pp. 4381-4395, 2012. Article (CrossRef Link)

[17] S. Jung and J. Lee, "Interference alignment and cancellation for the two-user X channels with a relay,” in Proc. of Personal Indoor and Mobile Radio Communications (PIMRC), 2013 IEEE 24th International Symposium on, IEEE, pp. 202-206, 2013. Article (CrossRef Link) 
[18] Y. Shu, Q. Wang, D. Shao and J. Zhang, "Interference neutralization and alignment in cognitive relay assisted 3-user interference channels," in Proc. of Vehicular Technology Conference (VTC Fall), 2014 IEEE 80th, pp. 1-5, 2014. Article (CrossRef Link)

[19] N. Lee and C. Wang, "Aligned interference neutralization and the degrees of freedom of the two-user wireless networks with an instantaneous relay," Communications IEEE Transactions on, vol. 61, no. 9, pp. 3611-3619, 2013. Article (CrossRef Link)

[20] W. Qiang, S. Yuquan, D. Minhua, X. Ji and T. Xiaofeng, "Degrees of freedom of 3-user MIMO interference channels with instantaneous relay using interference alignment,” KSII Transactions on Internet and Information Systems(TIIS), vol. 9, 2015. Article (CrossRef Link)

[21] N. Lee and R.W. Heath, "Degrees of freedom for the two-cell two-hop MIMO interference channel: interference-free relay transmission and spectrally efficient relaying protocol,” Information Theory, IEEE Transactions on, vol. 59, no. 5, 2013, pp. 2882-2896.

Article (CrossRef Link)

[22] Yanchun Li, Guangxi Zhu, Hua Chen, Minho Jo and Yingzhuang Liu, "SLNR-based user scheduling in multi-cell networks: from multi-antenna to large-scale antenna system, ” KSII Transctions on Internet and Information Systems, Vol.8, No.3 pp.945-964, March, 2014. Article (CrossRef Link)

[23] D. Wu, C. Yang, T. Liu and Z. Xiong, "Feasibility conditions for interference neutralization in relay-aided interference channel,” Signal Processing IEEE Transactions on, vol. 62, no. 6, pp. 1408-1423, 2014. Article (CrossRef Link)

[24] Q. Wang, Y. Dong, J. Zhao, N. Li, J. Qian and B. Liu, "Instantaneous relaying: Feasibility conditions for interference neutralization,” Communications Letters, IEEE, vol. 19, 2015. Article (CrossRef Link)

[25] R. Wang and X. Yuan, "MIMO multiway relaying with pairwise data exchange: A degrees of freedom perspective,” IEEE Transactions on Signal Processing, vol. 62, no. 20, pp.5294-5307, 2014. Article (CrossRef Link)

[26] D. Tse and P. Viswanath, Fundamentals of wireless communication. Cambridge, U.K.: Cambridge Univ. Press, 2005.

[27] I. Emre Telatar, Capacity of multi-antenna Gaussian channels, ATT,Bell Laboratories, Internal Tech. Memo., June 1995. Article (CrossRef Link) 


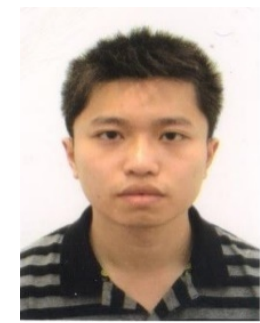

Bowei Zhang received his BS degree in from College of Communication Engineering, Chongqing University (CQU), Chongqing, China in June 2010. He is currently studying for his Ph.D. degree in communication and information system at College of Communication Engineering, CQU. His research interests include interference alignment, signal alignment and network coding in MIMO relay system.

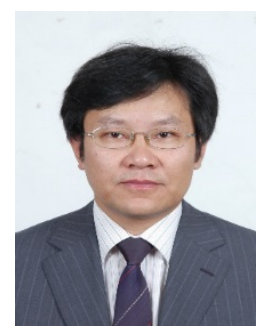

Wenjiang Feng received his Ph.D. degree in electrical engineering from Chongqing University in 2000. Currently, he is a professor at the college of communication engineering in Chongqing University. His research interests fall into the broad areas of communication theory, wireless communication.

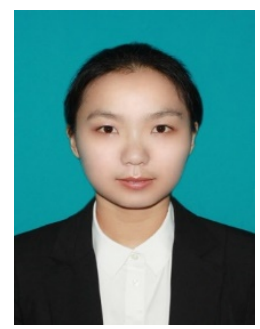

Tingting Dong received her BS degree in Chongqing University in June 2013. She is currently pursuing MS degree at College of Communication Engineering, CQU. Her research fields include wireless network communication and full-duplex communication.

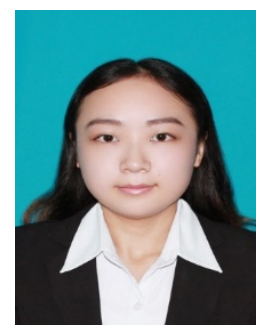

Yina Deng received her BS degree in Chongqing University in June 2013. She is currently pursuing MS degree at College of Communication Engineering, CQU. Her research fields include wireless network communication and interference alignment. 